2. van den Wildenberg FA, Houben PF, Maessen JG. Compartment-syndrome of the lower extremity after CABG. J Cardiovasc Surg (Torino). 1996;37:237-41.

3. Pasic M, Carrel T, Tonz M, Vogt P, von Segesser L, Turina M. Acute compartment syndrome after aortocoronary bypass. Lancet. 1993;341:897.
4. Scott JR, Daneker G, Lumsden AB. Prevention of compartment syndrome associated with dorsal lithotomy position. Am Surg. 1997;63: 801-6.

5. Tuckey J. Bilateral compartment syndrome complicating prolonged lithotomy position. Br J Anaesth. 1996;77:546-9.

\title{
Polydioxane (PDS) cord has insufficient reliability to securely close the sternum
}

\author{
Akihiko Usui, MD, Hideki Oshima, MD, Toshiaki Akita, MD, and Yuichi Ueda, MD, Nagoya, Japan
}

M edian sternotomy is a common approach to expose the heart for cardiac surgery. The sternum is usually closed with stainless wires or thick-braided sutures after surgery. We have used Polydioxane (PDS) cord sutures $1.0 \mathrm{~mm}$ in diameter for closing the sternum on 350 patients for the last one and a half year. But recently, three patients suffered broken PDS sutures and instability of the sternum several weeks after surgery. The sternum had to be closed immediately in two of them after the broken sutures because of wound dehiscence. One patient was observed closely without surgical intervention because of renal failure and MRSE carrier on skin culture.

\section{Cases}

All three patients had some risk factors for wound healing. One was a 73 -year-old man, who suffered an old myocardial infarction with poor left ventricle function, chronic atrial fibrillation and diabetes mellitus. He underwent on-pump 4-vessel aorto-coronary bypass grafting concomitant with Maze procedure. He suffered a minor stroke and required long mechanical ventilation therapy and tracheostomy on the $10^{\text {th }}$ postoperative day. Sternum instability was observed on the $12^{\text {th }}$ postoperative day after bronchoscopy. He revealed wound dehiscence on the $14^{\text {th }}$ day and sternum re-closure was performed on the same day. All 6 PDS cord sutures were broken beside the knot without any loose ligation or broken sternum.

One was a 75-year-old man with aortic arch aneurysm and angina pectoris. He had chronic obstructive pulmonary dysfunction and severe obesity. Total arch replacement was performed under hypothermic circulatory arrest concomitant with aorto-coronary bypass grafting. He was extubated on the second postoperative day and discharged from the intensive care unit on the third postoperative day.

\footnotetext{
Department of Cardiac Surgery, Nagoya University Graduate School of Medicine.

Corresponding author: Akihiko Usui, MD, Department of Cardiac Surgery, Nagoya University Graduate School of Medicine, Tsurumai 65, Showa-ku, Nagoya, Japan 466-8550 (Tel; 81-52-744-2376, FAX; 81-52-744-2383,

E-mail: ausui@med.nagoya-u.ac.jp).

J Thorac Cardiovasc Surg 2006;131:1174-5

$0022-5223 / \$ 32.00$

Copyright $\odot 2006$ by The American Association for Thoracic Surgery doi:10.1016/j.jtcvs.2005.12.043
}

Sternal instability was revealed on the $7^{\text {th }}$ postoperative day and wound dehiscence on the $9^{\text {th }}$ postoperative day. Reclosure of the sternum was performed on the $10^{\text {th }}$ postoperative day. Only the lower part of the sternum was broken, but all 6 PDS code sutures were broken without loose knot.

The last was a 68-year-old woman with angina pectoris. She had a history of atrial septal defect closure via right thoracotomy at 31 years of age. The case was complicated with asthma, diabetes mellitus and renal failure. Off pump 3 vessels coronary bypass grafting was performed. She was extubated on the next day and recovered well. But sternal instability occurred during an asthma attack on the $10^{\text {th }}$ postoperative day with a cracking sound. Her chest CT revealed a separation of several $\mathrm{cm}$ throughout the sternum. She was followed without sternal reclosure because she had no wound dehiscence, suffered from renal failure and MRSE carrier on her skin.

\section{Macroscopic Finding}

The broken PDS cord sutures of case 1 were carefully examined. All six PDS cords had completely snapped. Two of them were placed in the sternum manubrium and the other 4 were located just around the sternum body at the intercostal space. No loose knots were observed. Broken sites were 3 to $15 \mathrm{~mm}$ apart from the knot. The cord stumps gradually became thin, kinked with curled filaments and possibly had been torn off (Figures 1 and 2). Twenty $\mathrm{mm}$ away from the broken end on 2 of them, what appeared to be abrasion injures were observed. On the sternum manubrium where the cord protruded, the cord was thought to have been abraded on the edge of the bone.

\section{Discussion}

PDS cord suture is designed to close the sternum. It has enough strength for traction force, but it may be damaged by shear stress with a bone edge. The damaged part of the suture loses strength and may break under severe traction due to coughing or bronchial suction. Macroscopic findings of broken sutures indicated abrasion injury due to friction with the bone edge or that they were torn off under severe traction.

PDS cord is a synthetic absorbable suture. PDS cord of $1.0 \mathrm{~mm}$ in diameter has sufficient tensile strength $(180 \mathrm{~N})$ for closing the sternum before use. But it decreases gradually after use in the body. Manufacturer data show the tensile strength of $50-65 \%$ at 3 


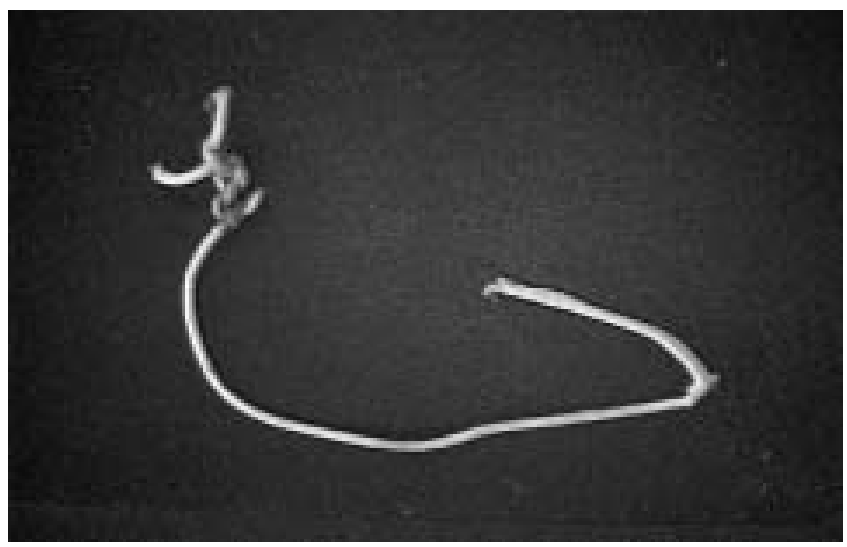

Figure 1. Low magnified image of broken PDS suture $(\times 5)$. No loose knot was observed. Broken sites were $3 \mathrm{~mm}$ apart from the knot. Another abrasion injury was observed $20 \mathrm{~mm}$ away from the broken site. One is the part of the cord just above the sternum, and the other is the part just below the sternum.

weeks and $30-40 \%$ at 6 weeks after implantation. This is another reason for broken sutures.

Broken sutures made of PDS cord may occur several weeks after surgery when patients have a persistent cough. PDS cord sutures do not have sufficient reliability to close the sternum, especially in patients with chronic obstructive pulmonary dysfunction and obesity. ${ }^{1}$

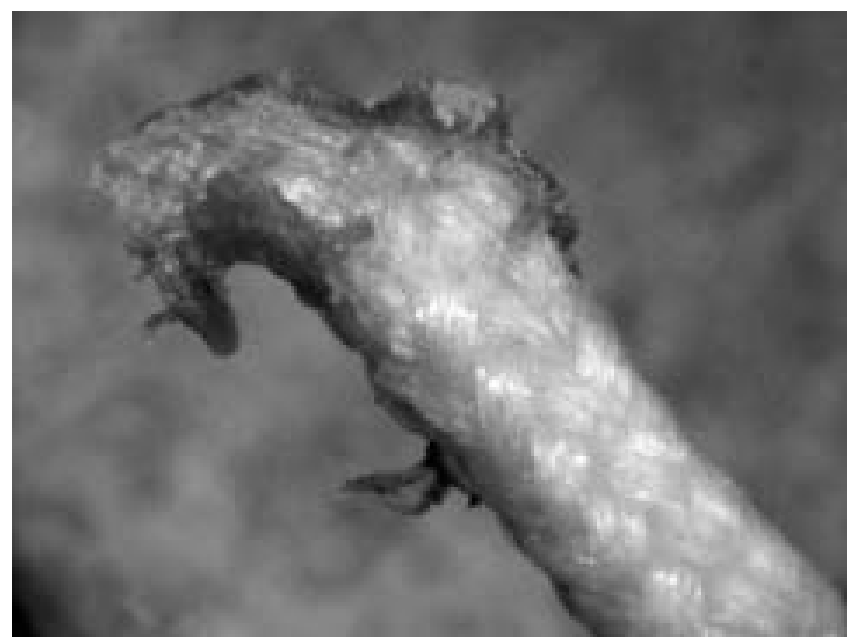

Figure 2. Magnified image of site of break (a) $(\times 60)$. The cord stump gradually became thin, kinked with curled filaments, and had possibly been torn off.

\section{Reference}

1. Ray JA, Doddi N, Regula D, Williams JA, Melverger, Polydioxane (PDS), a novel monofilament synthetic absorbable suture. Surgery, Gynecology \& Obstetrics 1981;153:497-507.

\section{Intraoperative finding of structural abnormality of bovine pericardial aortic prosthesis}

Uberto Da Col, MD, Davide Di Lazzaro, MD, Gino Di Manici, MD, Isidoro Di Bella, MD, and Temistocle Ragni, MD

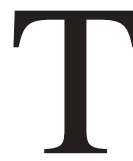

he bovine pericardial valve is an excellent valve, with a long, widespread use without reported intraoperative failures. ${ }^{1}$ However, 4 intraoperative structural failures of bovine pericardial bioprostheses placed in the mitral position were recently reported. ${ }^{2}$ The authors of this report hy-

From the S.C. di Cardiochirurgia, Azienda Ospedaliera di Perugia, Ospedale R. Silvestrini, Perugia, Italy.

Received for publication Nov 29, 2005; revisions received Dec 15, 2005; accepted for publication Dec 22, 2005.

Address for reprints: Davide Di Lazzaro, S.C. di Cardiochirurgia, Ospedale R. Silvestrini, S. Andrea delle fratte 06156 Perugia, Italy (E-mail: davide.dilazzaro@ospedale.perugia.it).

J Thorac Cardiovasc Surg 2006;131:1175-6

$0022-5223 / \$ 32.00$

Copyright $\odot 2006$ by The American Association for Thoracic Surgery doi:10.1016/j.jtcvs.2005.12.044 pothesized that the failures were caused by the distortion of the normal planar geometry of the prosthesis caused by mitral annular disease; this leads to failure of adequate central leaflet position and coaptation.

We report the intraoperative finding of a structural abnormality of the same type of bovine pericardial valve, detected after implantation in the aortic position, before closure of the aorta.

\section{Clinical Case}

A 69-year-old man underwent elective operation for aortic valve stenosis and dilatation of the ascending aorta. His heart had a poor global functionality, with an echocardiographic ejection fraction of $20 \%$ to $25 \%$. The operative approach was through median sternotomy; the ascending aorta appeared dilatated starting from the sinotubular junction up to $1.5 \mathrm{~cm}$ before the origin of the innominate artery. The Valsalva sinus and aortic arch appeared slightly dilated, and cardiomegaly (mainly for dilatation of the left ventricle) was observed. We opted for separate replacement of the aortic 